\title{
REINFORCEMENT LEARNING FROM SIMULATED ENVIRONMENTS: AN ENCODER DECODER FRAMEWORK
}

\author{
Benjamin Choo \\ Graham Crannel \\ Stephen Adams \\ Faraz Dadgostari \\ Peter A. Beling
Department of Engineering Systems and Environment
University of Virginia
Charlottesville, VA, UVA

\author{
Ann Bolcavage \\ Rolls-Royce Corporation \\ Indianapolis, IN, USA
}

\author{
Roy McIntyre \\ Rolls-Royce PLC \\ Derby, UK
}

\begin{abstract}
Reinforcement learning (RL) is used for sequential decision making such as operating and maintaining manufacturing systems. In RL, the system is modeled as a Markov decision process with states, actions, rewards, and policies. A policy is learned through repeated interaction with the environment. When a RL agent cannot interact with the real system due to time and cost constraints, a simulation of the system may be used in its place. Unfortunately, most simulations are not built for the purpose of interacting with a RL agent. Simulations built to function as the environment are often structured only according to the defined state, action, and reward and can lack fidelity, detail, and accuracy. We propose a general framework for bridging the worlds of simulation and RL. This is accomplished by placing "interpreters" between the simulation and the RL agent that translate information into a form that is coherent to each entity.
\end{abstract}

Keywords: reinforcement learning, Markov decision process, manufacturing.

\section{INTRODUCTION}

Reinforcement learning (RL) (Sutton and Barto 1998) is a machine learning technique used in sequential decision making situations. Recently, the popularity of RL based algorithms has quickly expanded due to their ability to efficiently solve large problems. One popular example is the use of RL to construct a system for playing the game of Go (Silver et al. 2016). An RL agent interacts with a system, or the environment as it is called, by sending actions to the system and receiving state and reward information in return. The policy for optimizing the total expected reward is estimated through repeated interactive episodes with the environment. However, RL algorithms can require a large number of interactions with the environment, often on the order of hundreds of thousands or even millions, to learn the optimal policy. Therefore, time and cost lim- 
itations often prevent RL agents from freely interacting with a real-world system, such as a manufacturing line. In these cases, a simulation of the real-world system can be used in place of the environment.

Many sequential decision making problems are modeled using a Markov decision process (MDP) (Roijers et al. 2013) which is composed of states, actions, and rewards. With the environment in a particular state, an agent takes an action from a set of available actions, and then experiences a reward and a state transition, both of which may be stochastic. The agent's objective is to maximize expected discounted future rewards. If the components of an MDP are known, an optimal policy can be found using dynamic programming. However, there are situations where dynamic programming is not possible. It may be difficult or impossible to estimate some components of the MDP, e.g. the transition probabilities. Furthermore, even if the transition probabilities are known, the state space may be so large that it is impossible to solve for the optimal policy.

$\mathrm{RL}$ is often referred to as a set of model-free algorithms because they learn an optimal policy through repeated direct interaction with the environment. Recently, model-based approaches have been combined with model-free algorithms which learn estimates of the model parameters as the agent interacts with the environment (Chebotar et al. 2017, Xie et al. 2016). In another approach, discrete event simulations or other models of the environment can be used in place of the true environment when time or availability are constrained. For the RL agent to interact with a simulation as the environment and effectively find an optimal policy, the simulation must be expressed in the MDP paradigm of states, actions, and rewards.

In simulation research, structuring the simulation with an MDP model in mind is rarely a high priority or even considered. Most high-fidelity simulations consist of building blocks that are different from what the RL agent needs. There are numerous types of simulations, and their underlying components can vary drastically. Typically, they include system state variables, a simulation clock, event lists, statistical counters, library routines, and the main program (Law 2013). Formal methods to define states and events have been introduced in the simulation design literature (Schruben 1983, Sargent 1988), however the states defined through these methods do not address the action or reward of the system. In most RL literature involving simulations, the MDP or state definitions are assumed to be readily available, or the process is ad hoc. Simulations built for RL purposes fit the necessary format of an MDP model. However, simulations or models used in RL can lack the detail and fidelity compared to simulations used in system analysis. The simplified simulation may not capture the intricate inner workings of the system.

There have been attempts from the simulation side of research to use simulation while utilizing RL as a solution method. Shuhui Qu, Jie Wang, and Shivani (2016) applied RL to solve a dispatching problem in a manufacturing system. A simple simulation was built with key variables to be used directly as part of the state, action, and reward structure. Doltsinis, Ferreira, and Lohse (2014) applied RL for production ramp up optimization. Despite the existence of simulation tools, both previously cited works used a simplified model. For instance, Shuhui Qu, Jie Wang, and Shivani (2016) put in constraints, such as not considering machine breakdowns, to the model. Doltsinis, Ferreira, and Lohse (2014) somewhat successfully generalize the MDP model building process, but has the limitation of defining the model states based on a human operator's perception of pertinent information. The solution method may be valid in both cases, but the bridging process is not general. Any changes to the system, including adding variables, changing the state definition, or accounting for intricate transition dynamics, could require rebuilding the simulation. From a wider perspective, there has been an increased effort from the simulation side to incorporate machine learning and RL with simulation. Qi et al. (2018) used big data and machine learning for fault-diagnosis for a reciprocating compressor. Martinez-Gil, Lozano, and Fernández (2017) have used multi-agent reinforcement learning for simulating pedestrian behaviour. Ghouti (2016) used neural network approaches for mobility prediction in mobile ad hoc networks. However, a general framework for defining simulation features and variables in the appropriate RL paradigm of states, actions, and rewards is needed. 
In some applications, high-fidelity simulations may already exist. Constructing a new simulation for the sole purpose of interacting with a RL agent would be redundant and possibly a waste of resources. In these situations, the optimal solution is to utilize the existing simulation and have the RL agent interact with it. However, in order for this to be possible, there must be an intermediary or interpreter between the simulation and the RL agent to convert information passing between the two entities into the correct format.

We propose Simulation Encoding / Decoding for Reinforcement Learning (SEDR), a framework for converting high-fidelity simulation information into a format acceptable to a RL agent. The proposed framework is defined by its two primary procedures - decoding information from the simulation for the RL agent and encoding the actions of the RL agent for the simulation. The framework introduces a procedure for converting the variables in the simulation to states and rewards and for converting actions into control signals and routines. The Encoding and Decoding modules act as interpreters between the simulation and the RL agent. The focus of this research is not on building simulations for reinforcement learning, nor is it on reinforcement learning algorithms with simulation as the environment. The primary focus of this paper is to propose and demonstrate the usefulness of the SEDR framework, which, to the best of our knowledge, is the first formal framework for intermediate models between a simulation and a RL agent designed to interpret information. The SEDR framework aims to act as the intermediary between the two worlds of simulation research and reinforcement learning research. The authors would like to point out that Bonsai, a company recently acquired by Microsoft, claims it can perform a similar task where the linking of an RL agent with a simulation is performed automatically. However, there are requirements for the simulation such as being written in a particular language and having specific components. In contrast, SEDR is a general framework for linking any simulation with an RL agent.

The presented framework is quite general, but our example and evaluation is conducted using a discrete event simulation in the manufacturing domain. In manufacturing, discrete event simulations have found wide application for process design and improvement; though in recent years, their use has moved from process design to control decision (Negahban and Smith 2014). In our view, the fine grained control problems that are now arising in smart manufacturing environments present an ideal environment for application of RL utilizing simulation.

\section{REINFORCEMENT LEARNING}

RL (Sutton and Barto 1998) is an area of machine learning that focuses on an agent's sequential decision making. The problems involve tying states (of the world, simulation, environment, etc) to particular actions in a way that will maximize some notion of reward. An agent observes the current state of the environment, decides on an action, implements the action, and allows time to progress to the next decision point. The agent receives a reward signal and new state information from the environment. A key factor is that the agent is not directly told which action to take in a given situation; it discovers which actions are best by exploring, evaluating, and learning. This interaction is shown in Figure 1(a), where the agent is interacting with the true environment. In some cases, interacting with the true environment is not possible, so a simulation of the environment is used, as illustrated in Figure 1(b).

The goal of the agent is to devise a policy that maximizes expected future reward. For an infinite horizon problem, a discount factor $\gamma$ is used to represent the diminishing importance of future events with respect to the current decision. At time $t$, the sum of future rewards can be written as $G_{t}=\sum_{k=0}^{\infty} \gamma^{k} R_{t+k+1}$. When the evolution of the state follows the Markov property (the transition to the next state does not depend on the sequence of transitions that came before, i.e. the process is memoryless), the agent-environment interaction can be formulated as an MDP. An MDP is defined by the set of states $\mathscr{S}$, the set of available actions $\mathscr{A}$, the expected reward given the state and the action $\mathscr{R}_{s}^{a}=\mathbb{E}\left[R_{t} \mid S_{t}=s, A_{t}=a\right]$, and a state transition function $\mathscr{P}_{s s^{\prime}}^{a}=\mathbb{P}\left(S_{t+1}=s^{\prime} \mid S_{t}=s, A_{t}=a\right)$. A policy $\pi$ maps states to actions, where $\pi(a \mid s)$ represents the probability 


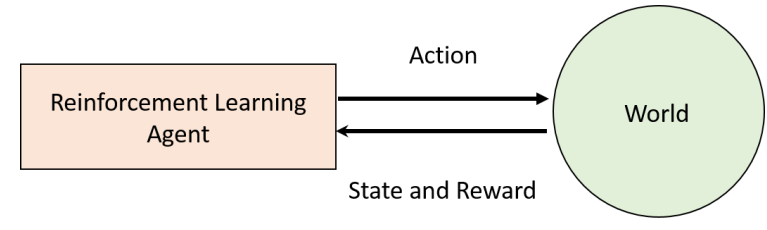

(a)

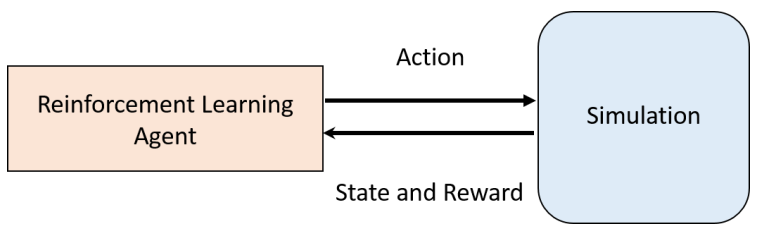

(b)

Figure 1: Reinforcement learning agents interacting with the (a) real environment and (b) a simulation of the real environment.

of taking action $a$ in state $s$. A major component of solving a RL problem is determining the value of being in a state. For MDPs, the value of following a certain policy in a certain state is given by the value function, $V_{\pi}(s)=\mathbb{E}_{\pi}\left[G_{t} \mid S_{t}=s\right]$. State-action pairs can also have a value, defined as $Q_{\pi}(s, a)=\mathbb{E}_{\pi}\left[G_{t} \mid S_{t}=s, A_{t}=a\right]$. The value function and the action-value function can be decomposed into

$$
V_{\pi}(s)=\sum_{a \in \mathscr{A}} \pi(a \mid s)\left(\mathscr{R}_{s}^{a}+\gamma \sum_{s^{\prime} \in \mathscr{S}} \mathscr{P}_{s s^{\prime}}^{a} V_{\pi}\left(s^{\prime}\right)\right)
$$

and

$$
Q_{\pi}(s, a)=\mathscr{R}_{s}^{a}+\gamma \sum_{s^{\prime} \in \mathscr{S}} \mathscr{P}_{s s^{\prime}}^{a} \sum_{a^{\prime} \in \mathscr{A}} \pi\left(a^{\prime} \mid s^{\prime}\right) Q_{\pi}\left(s^{\prime} \mid a^{\prime}\right)
$$

The objective in RL is to find an optimal policy $\pi^{*}$ which maximizes $V$ or $Q$

$$
V^{*}(s)=\max _{a}\left(\mathscr{R}_{s}^{a}+\gamma \sum_{s^{\prime} \in \mathscr{S}} \mathscr{P}_{s s^{\prime}}^{a} V^{*}\left(s^{\prime}\right)\right),
$$

and

$$
Q^{*}(s, a)=\mathscr{R}_{s}^{a}+\gamma \sum_{s^{\prime} \in \mathscr{S}} \mathscr{P}_{s s^{\prime}}^{a} \max _{a^{\prime}} Q^{*}\left(s^{\prime} \mid a^{\prime}\right)
$$

Methods for learning through interactions with the environment without an explicit MDP are collectively known as model-free RL (Sutton and Barto 1998). Parts of the MDP's structure, usually the transition probabilities or the reward, might be unavailable due to several reasons: a lack of data, the size of the state space, or inherent stochastic properties in the environment. Model-free RL is an effective method for optimizing sequential decision making in large scale environments. The recent success of Google and DeepMind's AlphaGo would not have been possible without model-free RL: if explicitly represented, the system would have required matrices with more than $10^{200}$ entries (Silver et al. 2016).

One model-free RL algorithm is Q-learning. In Q-learning, the actions are chosen using an $\varepsilon$-greedy approach, where with probability $\varepsilon$ an action is chosen at random and with probability $1-\varepsilon$ a greedy action is chosen using the previously estimated $Q$ values. The $Q$ values are updated at each step in the episode using

$$
Q(s, a)=Q(s, a)+\alpha\left[r+\gamma \max _{a} Q\left(s^{\prime}, a\right)-Q(s, a)\right],
$$




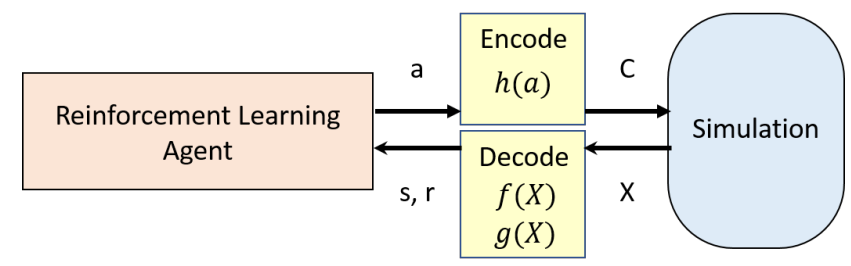

Figure 2: Reinforcement learning agents interacting with a simulation of the environment with SEDR framework in place. Action, control, state, reward, and simulation state, are represented by $a, c, s, r$, and $X$ respectively. The action encoding mapping, state decoding mapping, and reward decoding mapping, are represented by $h(a), f(X)$, and $g(X)$, respectively.

where $r$ represents the reward immediately received after taking action $a$ in state $s$.

In reinforcement learning, the agent makes decisions based on the state and reward from the environment. There have been studies using simulation as the environment for reinforcement learning. However, the specific approach for using simulation in reinforcement learning has been case by case. Our goal is to develop a generalized framework for implementing RL agents in simulation environments.

\section{METHOD}

We propose the SEDR framework for bridging the gap between the simulation structure, which consists of components such as system state variables, simulation clock, event list, statistical counters, library routines, and main program, and the RL structure, which consists of state, action, reward, and policy. The RL (Figure 1(b)) is further developed by adding the Encoding and Decoding procedures as shown in Figure 2. The Decoding procedure converts simulation variable values into RL states and rewards. The Encoding procedure encodes the action from the RL agent into control signals for the simulation. Once Encoding and Decoding are in place, an optimal policy can be learned through the use of RL methods such as Q-learning.

\subsection{Decoding Procedure}

The objective of the Decoding procedure is to select a mapping that would create the RL state and reward. The simulation variable values are used as inputs to create a corresponding state and reward outputs. The state of the system in a simulation is tracked by a collection of variables. For example, the state variables in a single-server queuing system include server status, number in queue, times of arrival, time of last event, etc. Let us define a vector Var as a collection of all variables $v$ in the simulation $\operatorname{Var}=\left[v_{1}, v_{2}, v_{3}, \ldots, v_{m}\right]$, where $m$ is the number of variables in the simulation. Different values of $v$ create different instances of Var which leads to different simulation states or $X$. The theoretical simulation state space consists of all possible combinations of each possible variable value. The size of the simulation state space $X$ becomes the product of possible values of all simulation variables. Though in practice many of the states will not be reached due to the inherent physical rules and characteristics of the system.

The objective of the Decoding procedure is to split the space of simulation state $X$ into mutually exclusive and collectively exhaustive subspaces, where each subspace represents a state or a reward of the system from the learning agent's perspective. In other words, the Decoding procedure partitions the space of simulation states into blocks where each block contains all the simulation states that are considered to be equivalent from the learning agent's perspective as depicted in Figure 3. 


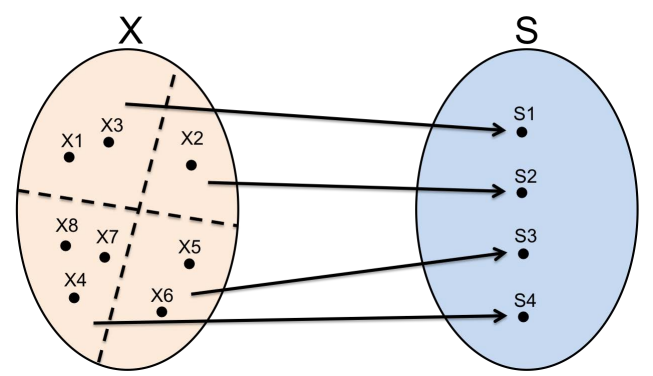

Figure 3: The state decoding process maps the simulation space $X$ into RL state $S$.

To be more formal, let us assume $E$ represents the equivalence relation defined on the space of simulation states. Accordingly, the set of all possible equivalence classes of $X$, denoted as $X / E:=\{[x] \mid x \in X\}^{1}$, formally called the quotient set of $X$ by $E$, defines the state space for the learning agent. Since every equivalence relation on $X$ is the equivalence kernel of a mapping $f: X \rightarrow X / E$, given $\mathscr{F}$ denoting the space of all surjective mappings defined on the space of simulation states (Lane and Birkhoff 1999, Birkhoff and Lane 2008), we can formulate the design of the Decoding procedure as finding an appropriate $f \in \mathscr{F}$ for simulation state decoding such that $f\left(X_{\tau}\right)=s_{t}$, where $X_{\tau} \in X$ is the value of the variables at simulation clock time $\tau$, and $s_{t}$ is the RL state at time $t$. How to define such partitioning mappings appropriately is out of the scope of this paper, but see Jackson et al. (2005) and Moore (1994).

When the Decoding process is applied to mapping simulation variables to states, the relationship between simulation state space and the state space in RL will have the following characteristics.

- State Exhaustiveness: All possible values of simulation variable Var are mapped to a state $s$.

- State Mutual Exclusiveness: For a particular value of simulation variable Var there is only one corresponding state $s$ in the RL space.

It is not always guaranteed that there will only be one way of defining the states. The decision of selecting a particular state definition over another must be considered as part of the design process. In other words, consideration must be put into finding the appropriate mapping out of several candidate mappings $f(X)$.

The next step in the Decoding procedure is to define $g(X)$, the mapping of simulation state $X$ in the simulation space to the reward of RL space. The reward $r$ is a scalar value produced by the environment when an action is taken and state change is made including self-transitions. It is highly dependent on the stakeholder's interest. For example, if the stakeholder would like to find the optimal policy for reducing production time per parts produced, then time related variables become an important ingredient, and other labor or material cost variables become less important in building $r$. As with the state decoding procedure, we formulate the design of the reward decoding procedure as finding an appropriate $g \in \mathscr{F}$ for simulation state decoding such that $g\left(X_{\tau}\right)=r_{t}$, where $X_{\tau} \in X$ is the value of the variables at simulation clock time $\tau$, and $r_{t}$ is the RL reward at time $t$. Therefore, the relationship between simulation state space and the reward space in RL will have the characteristics of being exclusive and exhaustive.

\footnotetext{
${ }^{1}[x]:=\{y \mid y \in X$ and $y E x\}$ represents the equivalence class $[x]$
} 


\subsection{Encoding Procedure}

The objective of the Encoding procedure is to define a mapping $h(a)$ that converts RL agent action $a$ into a vector of control signals $C$ to be consumed by the simulation. The set of control variables that make up $C$ are a subset of simulation variables.

In simulation, the system state changes when an event occurs. At the moment of the event, the corresponding event routine handles the event according to the current variable values. Such commands for changes to a set of control variables in simulation is different from actions in RL. The conversion mapping $h(a)$ matches the agent action $a$ into the corresponding vector of control signals $h\left(a_{t}\right)=C_{t}$.

The number of the control routines in the simulation space is greater or equal to the number of actions available in RL space. Thus, the mapping $l(X)$ is first defined from simulation space to RL space as with the Decoding procedure for state and reward. Once the mapping is defined, as a formality, the encoding $h(a)$ is defined as the inverse form of $l(X)=h^{-1}(C)=a$. The mapping $l(C)$ needs not be exhaustive. However, the inverse mapping $h(a)$ must be exhaustive. Certain control signal value vectors may not be of interest to the decision maker thus being excluded as a possible action to the decision maker. The mapping must only be mutually exclusive. A particular $X$, which includes the simulation state and the control sequence, may only be mapped to one action in the RL space. If a variable value combination $X$ that includes $C$ is mapped to more than two actions, it means the two actions may be combined to a single action in RL.

Once the Encoding and Decoding procedures are performed, the basis for applying RL algorithms is in place. Solution methods such as Monte Carlo Learning, SARSA Learning, SARSA $(\lambda)$, Q-Learning, and $\mathrm{Q}(\lambda)$-Learning are then used to learn the optimal decision policy.

\section{CASE STUDY}

The proposed SEDR framework is demonstrated on a manufacturing resource allocation example. Since there are multiple stations involved in the process, careful consideration must be put into the definition of the states. State definition for MDPs is often difficult and not straight forward (Scherer, Adams, and Beling 2018). The process under examination is known as thermal spray. It is one of many similar processes that are used to apply heat resistant coatings to certain areas of a blade for gas turbine engines. Since the coating is only required on specific areas of the part, each one must go through several stages of preparation and masking before they receive their final coating layer. The steps in the process are as follows: 1) initial masking to section off desired areas, 2) grit blasting to rough the surface of the metal, 3) masking for bond coat (first coating layer), 4) bond coat spraying, 5) re-masking for top coat, 6) top coat spraying, and 7) finishing and cleaning. Each station requires at least one worker to operate; grit blasting, bond coating, and top coating all require exactly one person. Parts arrive in batches of 32 and are put into 8 groups of 4 parts each. The blades must be processed through the bond coating or top coating as a single batch of 32 . The bond coating and top coating both use the same spraying booth, so they cannot be done at the same time. Transitioning from one coating type to the other requires booth downtime. At 10-minute intervals, the floor manager can reassign people from one station to another in order to improve the process flow and increase output. In the real world manufacturing process, they can re-assign whenever they want; but for the purposes of this simulation, it is necessary to discretize the decision points.

Since in this example it is possible to dynamically switch the station operators, the underlying mathematical problem is dynamic allocation optimization of a queuing system, which can be modeled and solved as a dynamic optimization problem over a queuing network or as an online parameter learning problem. We choose to use a simulation approach in combination with reinforcement learning to capture and optimize the dynamics of the queuing system through an adaptive allocation of operators to different stations in a 


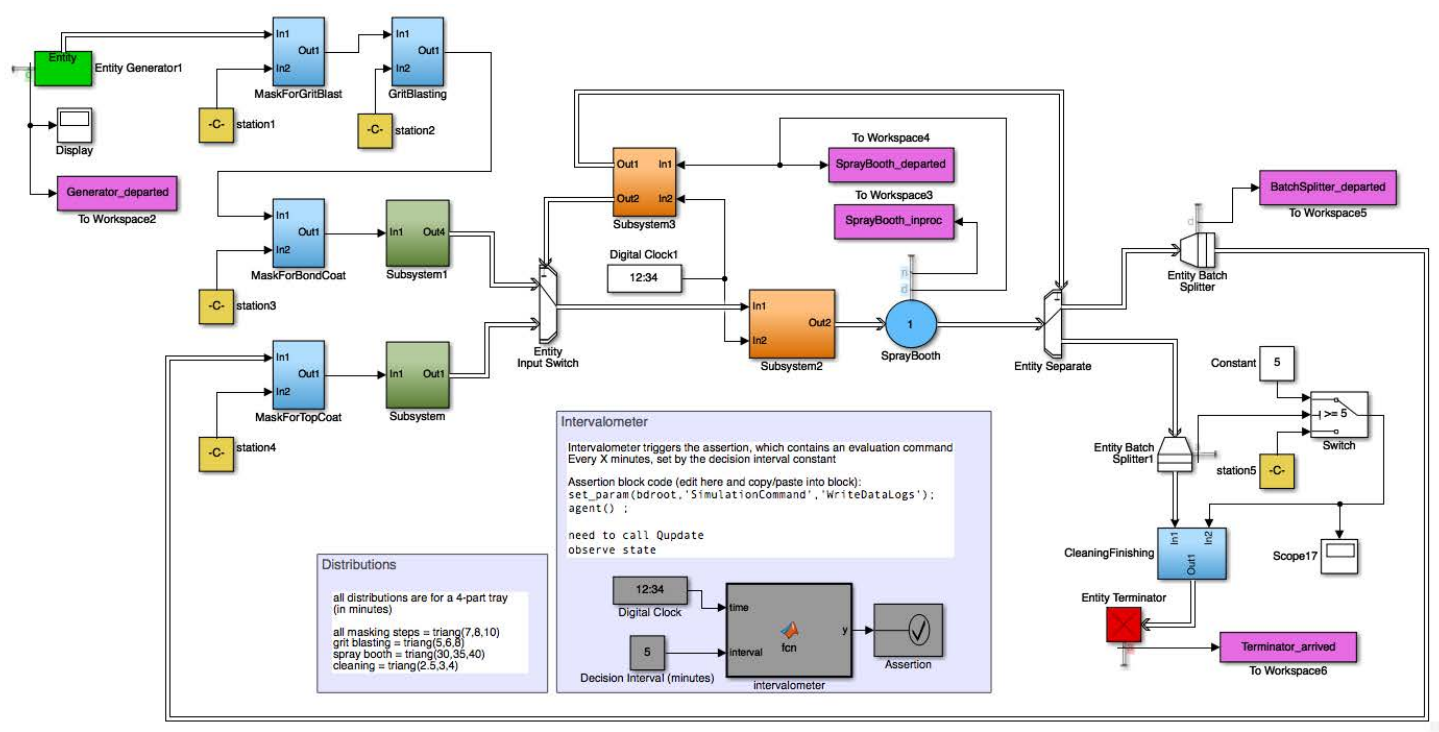

Figure 4: Simulation Diagram.

computationally efficient way. Also, this will provide us with a methodological approach to use already existing high-fidelity simulations, instead of developing a queuing optimization model from scratch.

The goal of the RL agent is to devise a policy that properly allocates workers at each of the stations so as to achieve the fastest throughput (essentially a dynamic resource allocation problem). This goal is achieved by modeling the reward function as minimizing the queue lengths at each station. The workers can be put at any of the stations and may be moved from station to station at will. The system is modeled as a variable time-step, discrete event simulation and is built using Mathworks' SimuLink software. Figure 4 shows a screenshot of the top-level model. It consists of multiple queues, servers (workers), gates, and conditional switches. The processing times for each worker at each station are stochastic and governed by triangular distributions that were estimated from data provided by an industry partner. Processing capacity at each station increases linearly with each worker.

The Decoding step is used to convert simulation states $X_{t}$ into RL state vectors $S_{t}$ that can be used by the RL agent. For this simulation, $X$ consists of queue lengths, worker allocation information, spray booth state, and completed part count. Only a portion of $X$ is necessary for the learning agent to make decisions, therefore the decoder creates the following state vector $X_{t}=\left(v_{1}, v_{2}, \ldots, v_{7}, b\right)$, where $v_{1}$ through $v_{7}$ are the lengths of the queues at each station (counted by part group, including the ones in-process) and $b$ is the state of the spraying booth (bond-coating or top-coating). In addition to state vectors, the Decoding step converts simulation variables $X$ into rewards $r_{t}$ as follows $r_{t}=g_{1}\left(f\left(X_{t}\right)\right)=g\left(s_{t}\right)=-\sum_{i=1}^{7} \omega_{i} v_{i}^{2}$, where $v_{i}$ is the queue length at station $i$, and where $\Omega$ is a vector of coefficients for each of the queues. In this case, $\omega_{i}$ is larger for small $i$ and decreases as $i$ increases: $\Omega=(6,5,4,3,2,1,1)$. This represents the fact that it is worse to have queues build up in the early stages of the process. This incentivizes the learning agent to allocate workers in order to move parts through the process as quickly as possible.

Essentially, the reward represents a quadratic holding cost summed over each of the process queues. The reward depends only on the current state, since re-allocating workers to different stations does not incur a cost (hence the compound function). As well as the holding cost described above, the agent gains a large bonus upon reaching the terminal state (i.e. when all queues are empty and when the completed part count equals the initial batch size) $r_{\text {terminal }}=g_{2}\left(X_{\text {terminal }}\right)=10^{5}$. This reward function is used only when the terminal conditions are met: when $X_{t}=X_{\text {terminal }}$. After the learning agent decodes the simulation variables 


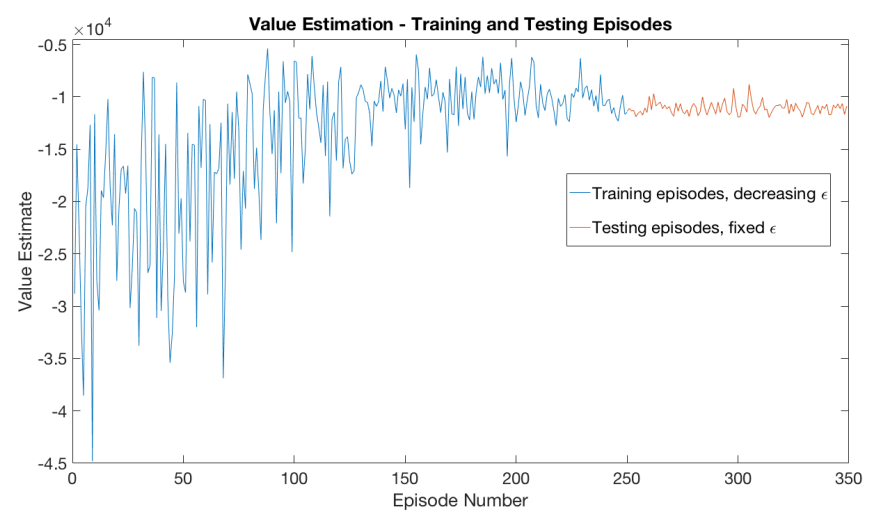

Figure 5: Value estimates for 250 training episodes followed by 100 testing episodes.
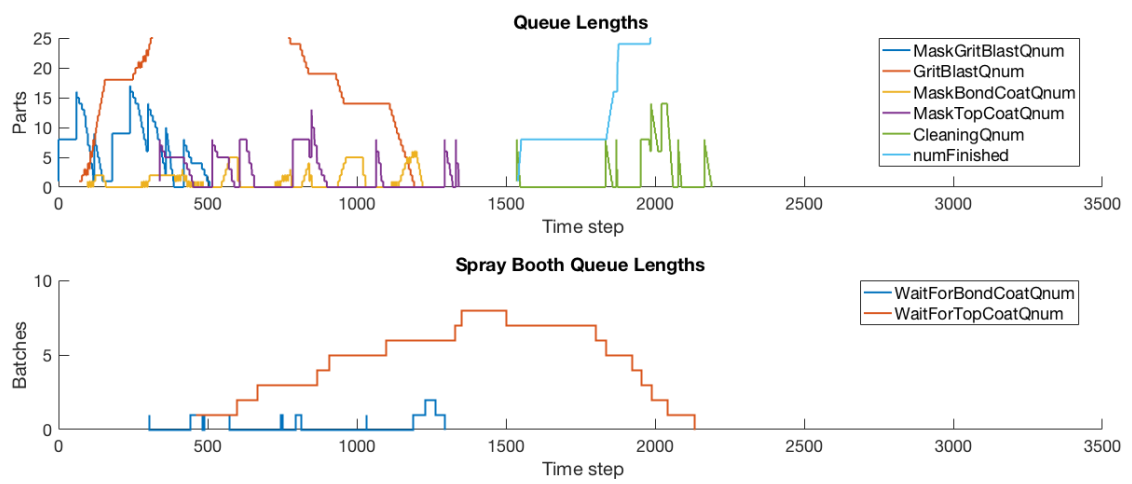

Figure 6: Queue lengths and spray-booth queue lengths - 1 episode of training.

into state vectors, it makes a decision as to what action should be taken. The decision is in the form of an action vector $A_{t}=\left(a_{1}, a_{2}, \ldots, a_{6}\right)$, where $\sum_{i=1}^{6} a_{i} \leq N$, and where $N$ is the total number of available workers. Actions $a_{1}$ through $a_{6}$ are the desired number of workers to be assigned to each of the 6 stations (the bondcoat and top-coat both happen at the same station, so only 6 stations physically exist). And, as mentioned before, grit blasting and the spray booth each have a maximum of 1 worker.

The chosen action vector $A$ is fed directly back into the simulation and the worker-allocation values are updated accordingly. Thus, the Encoding procedure is essentially a one-to-one mapping of actions to control signals. The control space is defined by the stakeholders and the physical limitations of the real-world system on which the simulation is based.

The Q-learning algorithm was used to estimate an optimal policy. The simulation was trained for 250 episodes with $\alpha=0.75, \gamma=0.9$, and $\varepsilon=1-i / N$, where $i$ is the episode number, and $N$ is the total number of training episodes (in this case $N=250$, so $\varepsilon$ decreases linearly from 1 to 0 over the course of the training period). After the training period, $\varepsilon$ was set to $1 / 250$ (so as to maintain some exploration) and the simulation was run for 100 episodes. Figure 5 shows the value function estimation over the course of the 250 training episodes and then the 100 testing episodes. The figure shows that the learner converges to a near-optimal policy. A better policy may be reached by modifying the learning rate and discount factor, but that is not within the scope of this paper and will be considered in future work. Figure 6 shows an example of queue length progression for the very first episode. The agent initially struggles with appropriately allocating workers to the grit blasting process. Figure 7 shows the queue progression for that same learner at the end of 

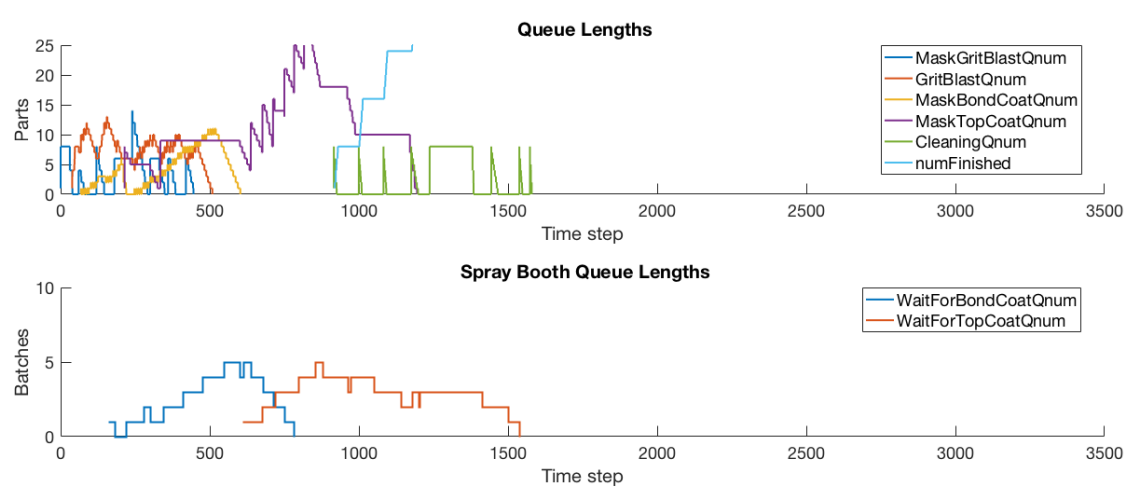

Figure 7: Queue lengths and spray-booth queue lengths - 250 episodes of training.

50 episodes. It is clear that the queue management has improved across the board (the x-axes are equivalent in Figures 6 and 7.)

\section{CONCLUSION}

The SEDR framework for converting high-fidelity simulation structure into MDP based structure for RL was presented in this paper. The methodology provides the structured approach in the format of Decoding and Encoding. The Decoding process maps simulation states to RL states and reward. The Encoding process maps RL actions to simulation control values. A real-world based manufacturing simulation was used as an example case for the application of SEDR. The SEDR framework is significant in the fact that it provides a general approach for defining states, actions, and rewards from simulation. In future work, the proposed methodology should be applied to more large-scale and complex simulations.

\section{ACKNOWLEDGMENTS}

The authors gratefully acknowledge funding provided by Rolls-Royce Corporation through its University Technology Center at the University of Virginia. The authors would also like to thank Turbine Service Technologies and, in particular, the thermal spray team and the process cell team.

\section{REFERENCES}

Birkhoff, G., and S. Lane. 2008. A Survey of Modern Algebra. Taylor \& Francis.

Chebotar, Y., K. Hausman, M. Zhang, G. Sukhatme, S. Schaal, and S. Levine. 2017. "Combining modelbased and model-free updates for trajectory-centric reinforcement learning". In Proceedings of the 34th International Conference on Machine Learning-Volume 70, pp. 703-711. JMLR. org.

Doltsinis, S., P. Ferreira, and N. Lohse. 2014, September. “An MDP Model-Based Reinforcement Learning Approach for Production Station Ramp-Up Optimization: Q-Learning Analysis". IEEE Transactions on Systems, Man, and Cybernetics: Systems vol. 44 (9), pp. 1125-1138.

Ghouti, L. 2016, August. "Mobility prediction in mobile ad hoc networks using neural learning machines". Simulation Modelling Practice and Theory vol. 66, pp. 104-121.

Jackson, B., J. D. Scargle, D. Barnes, S. Arabhi, A. Alt, P. Gioumousis, E. Gwin, P. Sangtrakulcharoen, L. Tan, and T. T. Tsai. 2005. "An algorithm for optimal partitioning of data on an interval". IEEE Signal Processing Letters vol. 12 (2), pp. 105-108.

Lane, S., and G. Birkhoff. 1999. Algebra. AMS Chelsea Publishing Series. Chelsea Publishing Company. 
Law, A. M. 2013. Simulation modeling and analysis. Fifth edition ed. McGraw-Hill series in industrial engineering and management science. Dubuque, McGraw-Hill Education.

Martinez-Gil, F., M. Lozano, and F. Fernández. 2017, May. "Emergent behaviors and scalability for multiagent reinforcement learning-based pedestrian models". Simulation Modelling Practice and Theory vol. 74, pp. 117-133.

Moore, A. W. 1994. "The parti-game algorithm for variable resolution reinforcement learning in multidimensional state-spaces". In Advances in neural information processing systems, pp. 711-718.

Negahban, A., and J. S. Smith. 2014. "Simulation for manufacturing system design and operation: Literature review and analysis". Journal of Manufacturing Systems vol. 33 (2), pp. 241-261.

Qi, G., Z. Zhu, K. Erqinhu, Y. Chen, Y. Chai, and J. Sun. 2018, January. "Fault-diagnosis for reciprocating compressors using big data and machine learning". Simulation Modelling Practice and Theory vol. 80, pp. 104-127.

Roijers, D. M., P. Vamplew, S. Whiteson, and R. Dazeley. 2013. "A survey of multi-objective sequential decision-making”. Journal of Artificial Intelligence Research vol. 48, pp. 67-113.

Sargent, R. G. 1988. "Event Graph Modelling for Simulation with an Application to Flexible Manufacturing Systems". Management Science vol. 34 (10), pp. 1231-1251.

Scherer, W. T., S. Adams, and P. A. Beling. 2018. "On the practical art of state definitions for Markov decision process construction". IEEE Access vol. 6, pp. 21115-21128.

Schruben, L. 1983, November. "Simulation modeling with event graphs". Communications of the ACM vol. 26 (11), pp. 957-963.

Shuhui Qu, Jie Wang, and G. Shivani. 2016, September. "Learning adaptive dispatching rules for a manufacturing process system by using reinforcement learning approach". In Emerging Technologies and Factory Automation (ETFA), 2016 IEEE 21st International Conference on, pp. 1-8, IEEE.

Silver, D., A. Huang, C. J. Maddison, A. Guez, L. Sifre, G. Van Den Driessche, J. Schrittwieser, I. Antonoglou, V. Panneershelvam, M. Lanctot, S. Dieleman, D. Grewe, J. Nham, N. Kalchbrenner, I. Sutskever, T. Lillicrap, M. Leach, K. Kavukcuoglu, T. Graepel, and D. Hassabis. 2016. "Mastering the game of Go with deep neural networks and tree search". Nature vol. 529 (7587), pp. 484-489.

Sutton, R. S., and A. G. Barto. 1998. Introduction to reinforcement learning, Volume 135. MIT Press Cambridge.

Xie, C., S. Patil, T. Moldovan, S. Levine, and P. Abbeel. 2016. "Model-based reinforcement learning with parametrized physical models and optimism-driven exploration". In 2016 IEEE International Conference on Robotics and Automation (ICRA), pp. 504-511. IEEE.

\section{AUTHOR BIOGRAPHIES}

BENJAMIN Y. CHOO is in the Ph.D program of the Engineering Systems and Environment (ESE) Department at the University of Virginia (UVA). He received his B.S. and M.S. degree from the Electrical Engineering Department at Yonsei University, Korea. He also received his M.E degree in Electrical Engineering from UVA. His research interests include prognostics and health management, smart manufacturing systems, machine learning, hierarchical reinforcement learning and deep reinforcement learning. His email is byc6j@virginia.edu.

GRAHAM T. CRANNEL received his Masters degree from the ESE Department at UVA. He received a B.A. in Physics from Austin College in Texas. He works in the Adaptive Decision Systems (ADS) Lab and his research interests include reinforcement learning, simulation, computer vision, and human factors. His email is gc4yd@virginia.edu. 
DR. STEPHEN ADAMS is a Principal Scientist in the ESE Department at UVA. He received his Ph.D. from the University of Virginia in Systems Engineering in December of 2015. His research interests include feature selection, machine learning with cost, sequential decision making, reinforcement learning, and probabilistic modeling of systems. He is currently part of the ADS Lab at UVA and his research is applied to several domains including activity recognition, prognostics and health management for manufacturing systems, psychology, cybersecurity, data trustworthiness, natural language processing, and predictive modeling of destination given user geo-information data. His email is sca2c@ virginia.edu.

FARAZ DADGOSTARI is a Ph.D. Candidate in the ESE Department at UVA working in ADS Lab and Financial Decisions Engineering research group. His research interests are in the theoretical and computational aspects of AI-supported sequential decision making in complex systems and adaptive decision making under systemic risk and uncertainty with emphasis on model-based data-driven approaches to systems design and assessment. His research on socio-economic and human-AI complex systems has found application in a variety of domains including knowledge acquisition systems, computation law, NLP, and mechanism design for financial, economic and smart energy systems. His email is fd4cd@virginia.edu.

DR. ANN BOLCAVAGE FASM is currently Engineering Associate Fellow at Rolls-Royce and leads the strategic development, validation, and deployment of critical coating materials and manufacturing technologies to support current and future aerospace gas turbine engines. Her areas of expertise include ceramic and metallic coating materials for hot-section engine components, the application of coating materials using thermal spray technology, and 'green' methods for coating manufacturing and repair. Dr. Bolcavage is an honors graduate of Lehigh University and received her advanced degrees in Metallurgical Engineering at the University of Wisconsin-Madison. She has co-authored 18 technical publications, given 23 conference / workshop presentations ( 5 invited), and has 13 patented inventions. Her email is ann.bolcavage@ rollsroyce.com.

ROY MCINTYRE is currently the thermal spray process lead at Rolls-Royce and leads the strategic development, validation, and deployment of the thermal spray process. He also supports the resolution of process issues at Rolls-Royce and within the supply chain, by developing novel solutions to process control issues. His areas of expertise are based around an extensive knowledge of all thermal spray processes and their use to deposit metallic and ceramic coatings for use both within the gas turbines, whilst at Rolls-Royce and also for general engineering applications, whilst working with Sulzer Metco and other supply chain organizations. Roy McIntyre is an honors graduate of Wolverhampton University having received a BSc (Hons) in Applied Sciences, based on Materials and Physics. Her email is roy.mcintyre@ rolls-royce.com.

DR. PETER A. BELING is Professor and Associate Chair for Research in the ESE Department at UVA. Dr. Beling's research interests are in the area of decision-making in complex systems, with emphasis on machine learning and adaptive decision support systems and on model-based approaches to system-of-systems design and assessment. His research has found application in a variety of domains, including missionfocused cybersecurity, reconnaissance and surveillance, prognostic and diagnostic systems, and financial decision making. He directs the UVA site of the Center for Visual and Decision Informatics, a National Science Foundation Industry/University Cooperative Research Center, and the ADS Lab, which focuses on data analytics and decision support in cyber-physical systems. Dr. Beling is the co-founder of the Financial Decision Engineering research group at UVA, which is a focal point for research on the mathematical modeling and risk management aspects of consumer and retail credit. Dr. Beling has served as editor and reviewer for many academic journals and has served as a member of five National Research Council panels. He received the Ph.D. in Operations Research from the University of California at Berkeley, M.S. in Operations Research from The George Washington University, and B.S. in Mathematics from the UVA. His email is pb3a@virginia.edu. 